\title{
Data Mining for Adverse Events of Tumor Necrosis Factor-Alpha Inhibitors in Pediatric Patients: Tree-Based Scan Statistic Analyses of Danish Nationwide Health Data
}

\author{
Viktor Wintzell ${ }^{1}$ (1) Henrik Svanström ${ }^{1,2} \cdot$ Mads Melbye $^{2,3,4} \cdot$ Jonas F. Ludvigsson $^{5,6,7,8} \cdot$ Björn Pasternak $^{1,2}$. \\ Martin Kulldorff ${ }^{9}$
}

Accepted: 7 October 2020 / Published online: 26 October 2020

(c) The Author(s) 2020

\begin{abstract}
Background and Objectives Tumor necrosis factor-alpha (TNF- $\alpha$ ) inhibitors are efficacious and considered generally safe in adults. However, pediatric-specific safety evidence is scarce. The aim of this study was to screen for signals of previously unknown adverse events of TNF- $\alpha$ inhibitors in pediatric patients.

Methods We conducted a data-mining study based on routinely collected, nationwide Danish healthcare data for 2004-2016. Using tree-based scan statistics to identify events with unexpectedly high incidence during TNF- $\alpha$ inhibitor use among patients with inflammatory bowel disease or juvenile idiopathic arthritis, two analyses were performed: comparison with episodes of no use and with other time periods from the same patient. Based on incident physician-assigned diagnosis codes from outpatient and inpatient visits in specialist care, we screened thousands of potential adverse events while adjusting for multiple testing.

Results We identified 1310 episodes of new TNF- $\alpha$ inhibitor use that met the eligibility criteria. Two signals of adverse events of TNF- $\alpha$ inhibitors, as compared with no use, were detected. First, there were excess events of dermatologic complications (ICD-10: L00-L99, 87 vs. 44 events, risk difference [RD] 3.3\%), which have been described previously in adults and children. Second, there were excess events of psychiatric diagnosis adjustment disorders (ICD-10: F432, 33 vs. 7 events, RD 2.0\%), which was likely associated with the underlying disease and its severity, rather than with the treatment. The selfcontrolled analysis generated no signal.

Conclusions No signals of previously unknown adverse events of TNF- $\alpha$ inhibitors in pediatric patients were detected. The study showed that real-world data and newly developed methods for adverse events data mining can play a particularly important role in pediatrics where pre-approval drug safety data are scarce.
\end{abstract}

\section{Key Points}

Based on screening of thousands of diagnoses from nationwide Danish health registers, we identified no signals of previously unknown adverse events of TNF- $\alpha$ inhibitors in pediatric patients.

Surveillance of adverse events from routinely collected real-world data can complement other analyses in generating pediatric-specific drug-safety evidence.

Electronic supplementary material The online version of this article (https://doi.org/10.1007/s40261-020-00977-5) contains supplementary material, which is available to authorized users.

Viktor Wintzell

viktor.wintzell@ki.se

Extended author information available on the last page of the article

\section{Introduction}

Tumor necrosis factor-alpha (TNF- $\alpha$ ) inhibitors have revolutionized the treatment of chronic inflammatory diseases and become increasingly common in children [1-3]. Previous studies in adults have found associations between TNF- $\alpha$ inhibitors and increased risk of adverse events, including serious infections and malignancies [4, 5]. However, extrapolation of adult data to children is not necessarily relevant, as has been shown regarding infections [6]. The pediatricspecific safety evidence for TNF- $\alpha$ inhibitors is generally scarce.

Detection of potential adverse events post-market approval is key to ensure safe use of drugs. Signals of previously unknown adverse events can be detected when new drugs are used at a larger scale and by a wider range of patients in clinical practice. Adverse event screening can play a particularly important role in pediatrics, where output 
of both clinical and observational studies is low $[7,8]$. To support optimal prescribing in children there is a need for pediatric-specific safety data $[9,10]$.

Spontaneous reporting systems have traditionally been the leading source of timely safety data [11]. However, due to increasing availability of large amounts of secondary data, including healthcare registers, new opportunities for signal generation have emerged [12]. The use of detailed patient data that are routinely collected over time enables detection of rare adverse events and decreases the risk of reporting bias and confounding.

The aim of this data-mining study was to screen for new signals of adverse events of TNF- $\alpha$ inhibitors in pediatric patients with inflammatory bowel disease (IBD) or juvenile idiopathic arthritis (JIA), applying newly developed methods for adverse events data mining on nationwide Danish health registers.

\section{Method}

\subsection{Study Population}

The study was performed based on Danish populationbased registers, linked via unique personal identity numbers. The source population was defined as all individuals living in Denmark aged $<18$ years at some time during the study period, 2004-2016. From the source population, we identified individuals with confirmed pediatric IBD or JIA, which was defined as at least two contacts with specialist care (inpatient or outpatient) with a physician-assigned IBD or JIA diagnosis during the study period or previously (1986-2016). These made up the study cohort of eligible individuals. See details in Supplementary Table 1 (Online Supplementary Material, OSM).

\subsection{Exposure Episodes}

From the study cohort, we identified episodes of followup of new TNF- $\alpha$ inhibitor use and episodes of no use of TNF- $\alpha$ inhibitors. New use of TNF- $\alpha$ inhibitors was defined as initiation of these biologics with no use within 2 years before. The TNF- $\alpha$ inhibitor episodes continued as long as the patient was on treatment. Treatment discontinuation was identified based on assumed duration of each drug administration (Supplementary Table 1, OSM) and an allowed gap in coverage (grace period) of a maximum of 90 days. Maximum length of follow-up was 3 years (see examples of the identification of episodes in Supplementary Fig. 1, OSM). Use of TNF- $\alpha$ inhibitors was defined based on procedure codes from the Danish National Patient Register (anatomical therapeutic chemical classification system [ATC] code L04AB). Biologic therapy is only administered in specialist care in Denmark and without incurring any cost for the patient [13].

Follow-up time with no exposure to TNF- $\alpha$ inhibitors in the last 2 years was considered no-use time. The no-use time was divided into episodes of a maximum of 3 years, which served as comparator episodes. No-use episodes were censored at initiation of TNF- $\alpha$ inhibitors. The episode design allowed individuals to be included in the study multiple times, as both TNF- $\alpha$ inhibitor and no-use episodes. All episodes were mutually exclusive; no time nor outcome event was counted more than once.

We performed two analyses: first, a propensity scorematched analysis where TNF- $\alpha$ inhibitor episodes were compared with no-use episodes; second, a self-controlled analysis where temporal risk windows during follow-up were compared within TNF- $\alpha$ inhibitor initiators.

\subsection{Propensity-Score Matching}

In the propensity score-matched analysis, TNF- $\alpha$ inhibitor and no-use episodes were matched on underlying disease (JIA, Crohn's disease [CD], or ulcerative colitis [UC; including unclassified IBD]) and on propensity scores. One patient could contribute multiple episodes to the analysis, but episodes in each matched pair had to come from different individuals. General potential confounders were included in the propensity-score model: demographics (age, sex), socioeconomic factors (family income and education level of parents), disease duration, drug use in the last year (oral corticosteroids and immunomodulators [thiopurines or methotrexate]), and general healthcare and drug use (number of prescription drugs, outpatient contacts, and inpatient admissions). We used a nearest-neighbor greedy matching algorithm (1:1 matching) with a caliper corresponding to two standard deviations of the log-odds of the propensity score [14]. The caliper was chosen to ensure that all TNF- $\alpha$ inhibitor episodes were matched and included in the analysis.

\subsection{Eligibility and Censoring}

Episodes were excluded if any of the following criteria were met at index: age $\geq 18$ years, patient lived outside of Denmark in the last 5 years, no specialist-care contact with IBD or JIA diagnosis in last 3 years, and use of any biologic in the last year (see Supplementary Table 1, OSM). All patients were censored at maximum follow-up (3 years), end of study period (31 December 2016), emigration, or death. TNF- $\alpha$ inhibitor users were also censored at treatment discontinuation and no-use episodes were censored at initiation of TNF- $\alpha$ inhibitors, if any. Additionally, within the matched pairs of the propensity score-matched analysis the episode with longer follow-up was censored at the end of follow-up for its match to make follow-up equal within matched pairs. 


\subsection{Adverse Events Data Mining}

We screened for adverse events based on physician-assigned diagnosis codes (10th revision of the International Statistical Classification of Diseases and Related Health Problems [ICD-10]) from outpatient and inpatient visits in specialist care. All ICD-10 codes as well as groups of related codes at three higher levels were evaluated as potential adverse events: disease chapters (e.g., I00-99 Diseases of the circulatory system), disease blocks (e.g., I10-15 Hypertensive diseases), and three- to four-position codes. As such, the ICD-10 codes define a structured tree of diagnoses and each grouping is defined by a cut on that tree. Diagnoses obtained from the register were recorded at the three- and four-position levels, which also represented individual cuts (see a detailed example in Supplementary Fig. 2, OSM). Codes that were not considered relevant as potential adverse events were excluded from the analysis, for example, congenital conditions, pregnancies and other codings unlikely to be caused by drugs (see Supplementary Table 2, OSM).

In the propensity score-matched analysis, we screened the data for cuts with a higher incidence in the TNF- $\alpha$ inhibitor episodes in comparison with the no-use episodes. In the self-controlled analysis, we screened for temporal clustering of potential adverse events following initiation of TNF- $\alpha$ inhibitors, that is, events with higher risk during certain time windows. Hence only the TNF- $\alpha$ inhibitor episodes were included in the self-controlled analysis.

Only incident events were considered for the analysis. In the propensity score-matched analysis, a code was incident if it was not preceded by the same code at the three-position level (e.g., I11.0 not preceded by any code starting with I11) at any time point before index to avoid inclusion of repeated events within individuals. In the self-controlled analysis, a look-back of 3 years in relation to the date of each event was used to determine if it was incident. Hence, the look-back was constant over follow-up. All events were analyzed but signals based on fewer than three exposed events could not be presented due to Danish data protection legislation.

\subsection{Tree-Based Scan Statistics}

To identify cuts with a higher incidence we used tree-based scan statistics, which are disproportionality statistics that adjust for multiple testing and that allow for simultaneous testing of diagnosis codes at all levels of granularity, that is, all cuts on the ICD-10 tree [15]. We screened for potential adverse events in the propensity score-matched analysis using the unconditional Bernoulli model [16, 17]. Exposure was assumed to follow a Bernoulli probability distribution. Under the null hypothesis and given the 1:1 matching ratio, events in all cuts were equally probable (probability $=0.5$ ) to occur during TNF- $\alpha$ inhibitor episodes as during no-use episodes. The alternative hypothesis was that events in at least one cut had a higher risk (probability $>0.5$ ) of occurring during TNF- $\alpha$ inhibitor episodes.

In the self-controlled analysis, we used the conditional tree-temporal scan statistic $[18,19]$. The analysis was conditioned on the total number of events over follow-up in each cut. Under the null hypothesis, events were uniformly distributed over follow-up. The alternative hypothesis was that there was at least one cut where the risk was higher in at least one of the analyzed risk windows. An advantage of this method is that no predefined risk windows are needed; temporal screening is performed over the entire follow-up period. We analyzed all unique, temporal risk windows of 2 days-1.5 years that fit during the maximum follow-up of 3 years (maximum window length was half of maximum follow-up). No window was shorter than $20 \%$ of the followup day it ended (e.g., a window that ended on day 100 was 20 days or longer) to avoid analyzing short-risk windows a long time after drug initiation.

Log likelihood ratios (LLRs) were calculated for each cut in the propensity score-matched analysis and for each cut-risk window in the self-controlled analysis. Inference was based on Monte Carlo simulation because there is no simple expression for the sample distribution of the LLRs [20]. $p$ values were obtained for each analysis by ranking the LLRs of the most likely cuts in relation to maximum LLRs simulated under the null hypotheses. Cuts with a $p$ value below 0.05 were considered significant. The analysis was performed with the free TreeScan v1.4 software (https:// www.treescan.org) and SAS v9.4 (SAS Institute Inc.).

\section{Results}

\subsection{Episode Characteristics}

During the study period, 1310 new users of TNF- $\alpha$ inhibitors were identified. Following 1:1 propensity-score matching, a cohort of 1310 pairs of TNF- $\alpha$ inhibitor episodes and no-use episodes was included. Episodes were well balanced on all variables, despite the large caliper used in the matching algorithm (Table 1). Of the TNF- $\alpha$ inhibitor episodes, $59 \%$ were female and mean (SD) age was 13.4 (4.0) years. The indication for TNF- $\alpha$ inhibitor use was JIA in $51 \%$ of the episodes, CD in $35 \%$, and UC in $14 \%$. Episodes were censored at the shortest length of follow-up within the matched pairs. Mean (SD) length of follow-up was 1.0 (0.9) years. For the self-controlled tree-temporal analysis, 1310 episodes of new TNF- $\alpha$ inhibitor use were included. The mean (SD) length of follow-up for these episodes was $1.2(0.9)$ years. 
Table 1 Characteristics of episodes of tumor necrosis factor alpha (TNF- $\alpha$ ) inhibitor use and no use included in unmatched and propensity score-matched cohorts

\begin{tabular}{|c|c|c|c|c|c|c|}
\hline \multirow[t]{2}{*}{ Characteristic } & \multicolumn{3}{|l|}{ Unmatched cohort } & \multicolumn{3}{|c|}{ Propensity score-matched cohort } \\
\hline & $\begin{array}{l}\text { TNF- } \alpha \text { inhibitors } \\
(n=1310)\end{array}$ & $\begin{array}{l}\text { No use } \\
(n=12,307)\end{array}$ & $\begin{array}{l}\text { Standardized } \\
\text { difference, \% }\end{array}$ & $\begin{array}{l}\text { TNF- } \alpha \text { inhibitors } \\
(n=1310)\end{array}$ & $\begin{array}{l}\text { No use } \\
(n=1310)\end{array}$ & $\begin{array}{l}\text { Standardized } \\
\text { difference, } \%\end{array}$ \\
\hline Male, no. (\%) & $532(40.6)$ & $5218(42.4)$ & 3.6 & $532(40.6)$ & $515(39.3)$ & 2.6 \\
\hline \multicolumn{7}{|l|}{ Age, no. $(\%)$} \\
\hline $0-12$ years & $480(36.6)$ & $5743(46.7)$ & 20.4 & $480(36.6)$ & $476(36.3)$ & 0.6 \\
\hline $13-15$ years & $381(29.1)$ & $3567(29.0)$ & 0.2 & $381(29.1)$ & $403(30.8)$ & 3.7 \\
\hline $16-17$ years & $449(34.3)$ & $2997(24.4)$ & 21.9 & 449 (34.3) & 431 (32.9) & 2.9 \\
\hline \multicolumn{7}{|c|}{ Disposable family income, no. (\%) } \\
\hline 1st quartile & $288(22.0)$ & $3116(25.3)$ & 7.9 & $288(22.0)$ & $302(23.1)$ & 2.6 \\
\hline 2nd quartile & $324(24.7)$ & $3066(24.9)$ & 0.4 & $324(24.7)$ & $320(24.4)$ & 0.7 \\
\hline 3rd quartile & $348(26.6)$ & $3071(25.0)$ & 3.7 & $348(26.6)$ & $353(26.9)$ & 0.9 \\
\hline 4th quartile & $350(26.7)$ & $3054(24.8)$ & 4.4 & $350(26.7)$ & $335(25.6)$ & 2.6 \\
\hline \multicolumn{7}{|l|}{ Parental education, no. (\%) } \\
\hline$\leq 9$ years & $91(6.9)$ & $1010(8.2)$ & 4.8 & $91(6.9)$ & $85(6.5)$ & 1.8 \\
\hline $10-12$ years & $619(47.3)$ & $5568(45.2)$ & 4 & $619(47.3)$ & $634(48.4)$ & 2.3 \\
\hline$\geq 13$ years & $600(45.8)$ & $5729(46.6)$ & 1.5 & $600(45.8)$ & $591(45.1)$ & 1.4 \\
\hline \multicolumn{7}{|l|}{ Calendar year, no. $(\%)$} \\
\hline 2004-2008 & $279(21.3)$ & $4727(38.4)$ & 38.1 & $279(21.3)$ & $544(41.5)$ & \\
\hline 2009-2013 & $572(43.7)$ & $4713(38.3)$ & 10.9 & $572(43.7)$ & $469(35.8)$ & \\
\hline 2014-2016 & $459(35.0)$ & $2867(23.3)$ & 26.1 & $459(35.0)$ & 297 (22.7) & \\
\hline JIA, no. (\%) & $673(51.4)$ & $8203(66.7)$ & 31.4 & $673(51.4)$ & $673(51.4)$ & \\
\hline Crohn's disease, no. (\%) & $457(34.9)$ & $1888(15.3)$ & 46.3 & 457 (34.9) & 457 (34.9) & \\
\hline Ulcerative colitis, no. (\%) & $180(13.7)$ & $2216(18.0)$ & 11.7 & $180(13.7)$ & $180(13.7)$ & \\
\hline \multicolumn{7}{|l|}{ Disease duration, no. (\%) } \\
\hline$<0.5$ years & $430(32.8)$ & $5315(43.2)$ & 21.5 & $430(32.8)$ & $446(34.0)$ & 2.6 \\
\hline$\geq 0.5$ years & $880(67.2)$ & $6992(56.8)$ & 21.5 & $880(67.2)$ & $864(66.0)$ & 2.6 \\
\hline Oral corticosteroids, no. (\%) & $594(45.3)$ & 1459 (11.9) & 79.8 & $594(45.3)$ & $543(41.5)$ & 7.9 \\
\hline $\begin{array}{l}\text { Immunomodulators (thiopu- } \\
\text { rines or methotrexate), no. }\end{array}$ & $720(55.0)$ & $1597(13.0)$ & 98.9 & $720(55.0)$ & $668(51.0)$ & 8 \\
\hline \multicolumn{7}{|c|}{ No. of prescription drugs in last year, no. (\%) } \\
\hline $0-1$ & $127(9.7)$ & 4747 (38.6) & 71.7 & $127(9.7)$ & $128(9.8)$ & 0.3 \\
\hline $2-5$ & $751(57.3)$ & $6037(49.1)$ & 16.6 & $751(57.3)$ & $765(58.4)$ & 2.2 \\
\hline $6+$ & $432(33.0)$ & $1523(12.4)$ & 50.8 & $432(33.0)$ & $417(31.8)$ & 2.4 \\
\hline \multicolumn{7}{|c|}{ No. of outpatient hospital contacts in last year, no. } \\
\hline $0-1$ & $43(3.3)$ & $3491(28.4)$ & 73.2 & $43(3.3)$ & $34(2.6)$ & 4.1 \\
\hline $2-4$ & $218(16.6)$ & $4738(38.5)$ & 50.4 & $218(16.6)$ & 234 (17.9) & 3.2 \\
\hline $5+$ & $1049(80.1)$ & $4078(33.1)$ & 107.5 & $1049(80.1)$ & $1042(79.5)$ & 1.3 \\
\hline \multicolumn{7}{|c|}{ No. of inpatient admissions in last year, no. (\%) } \\
\hline 0 & $400(30.5)$ & $7482(60.8)$ & 63.8 & $400(30.5)$ & $424(32.4)$ & 3.9 \\
\hline $1-2$ & $525(40.1)$ & $3529(28.7)$ & 24.2 & $525(40.1)$ & $516(39.4)$ & 1.4 \\
\hline $3+$ & $385(29.4)$ & $1296(10.5)$ & 48.6 & $385(29.4)$ & $370(28.2)$ & 2.5 \\
\hline
\end{tabular}

JIA juvenile idiopathic arthritis

\subsection{Propensity Score-Matched Analysis}

In the propensity score-matched cohort, 1284 incident, unique cuts of the ICD-10 tree were recorded during follow-up among all episodes. There were five cuts with a significantly high number of events in the TNF- $\alpha$ inhibitor episodes in comparison with the no-use episodes (Table 2). Two of the cuts were dermatologic: ICD-10 chapter Diseases of the skin and subcutaneous tissue (L00-99; 87 vs. 44 events; risk difference [RD] 3.3\%; $p$ value 0.017 ) and the 
related sub-branch, Dermatitis and eczema (L20-30; 34 vs. 8 events; RD 2.0\%; $p$ value 0.004$)$. For context, the excess number of events in the chapter L00-99 were also driven by disorders of skin appendages (L60-75; 28 vs. 10 events; $p$ value 0.39 ), papulosquamous disorders (L40-45; 10 vs. $<3$ events; $p$ value 0.43 ), and other disorders of the skin and subcutaneous tissue (L80-99; 13 vs. 10 events; $p$ value 1.00 ) (Supplementary Table 3, OSM). The other three significant cuts were ICD-10 block Anxiety, dissociative, stress-related, somatoform, and other nonpsychotic mental disorders (F40$48 ; 39$ vs. 11 events; RD 2.1\%; $p$ value 0.007 ), Reaction to severe stress and adjustment disorders (F43; 35 vs. 9 events; RD 2.0\%; $p$ value 0.008), and Adjustment disorders (F432; 33 vs. 7 events; RD 2.0\%; $p$ value 0.002) (Table 2 and Supplementary Table 3, OSM).

\subsection{Self-Controlled Analysis}

The self-controlled analysis was performed on the TNF- $\alpha$ inhibitor episodes. In total, 1036 unique cuts with incident events during these episodes were identified. No combinations of cuts and risk windows with significantly high incidence were identified. Hence, there were no signals of events with temporal clustering during follow-up.

\section{Discussion}

In this data-mining study of adverse events of TNF- $\alpha$ inhibitors in pediatric patients based on the nationwide Danish population, we found no signals of previously unknown adverse events. A signal of dermatologic complications that has been previously described in adults and children was detected, including excess cases of diseases of the skin and subcutaneous tissue, and dermatitis and eczema [21-26]. A detected signal of psychiatric diagnoses of anxiety, dissociative, stress-related, somatoform, and other nonpsychotic mental disorders, including reaction to severe stress and adjustment disorders, was likely associated with the underlying disease and its severity, rather than with the treatment. The study shows the utility and advantages of newly developed methods for adverse event data mining to generate safety information that is specific to children based on Scandinavian health registers.

Previous studies have described dermatologic adverse events of TNF- $\alpha$ inhibitor use. In particular, studies have described that new-onset psoriasis is a paradoxical adverse event of TNF- $\alpha$ inhibitors in patients with rheumatic disease and IBD. In adult IBD, dermatologic events have been recorded in 21-29\% (sample size $n=583-732$ ) of patients initiating TNF- $\alpha$ inhibitors, where median follow-up was $3-4.4$ years $[21,22]$. Psoriasis and cutaneous infections were the most common manifestations. In pediatric patients, one study found the risk of dermatologic events to be $11 \%$ $(n=409)$, with psoriasis, infections, and eczema being the most common diagnoses [23]. A small pediatric case series estimated the risk at $48 \%(n=84)$, where half of the patients with events had lesions that were considered severe [26]. The risk of new-onset psoriasis among pediatric TNF- $\alpha$ inhibitor users has been estimated at $8-14 \%(n=73-409)$ [23-25]. In our analysis, $6.6 \%$ of TNF- $\alpha$ inhibitor episodes had at least one incident event in the chapter Diseases of the skin and subcutaneous tissue (L00-99) and the risk difference in comparison with no use was $3.3 \%$.

The previous pediatric studies are one-arm case series or cohort studies that do not estimate the risk in relation to nontreated patients, that is, the relative risk or risk difference. To inform clinical practice about the potential dermatologic risks in pediatric patients, pharmacoepidemiologic studies in large, unselected populations with suitable comparators are needed, since clinical trials of suitable power will unlikely be conducted.

Our analysis also generated a signal of adjustment disorders (F432), which is part of reaction to severe stress and adjustment disorders (F43), and the ICD-10 block Anxiety, dissociative, stress-related, somatoform and other nonpsychotic mental disorders (F40-48). A plausible interpretation is that the signal reflects an association with the burden of

Table 2 Cuts on the ICD-10 tree with a significantly high risk in tumor necrosis factor-alpha (TNF- $\alpha$ )-inhibitor episodes as compared with nouse episodes from the propensity score-matched analysis

\begin{tabular}{llccc}
\hline Cut (ICD-10 code) & $\begin{array}{l}\text { Exposed events } \\
\text { (TNF- } \alpha \text { inhibitor) }\end{array}$ & $\begin{array}{l}\text { Unexposed } \\
\text { events (no use) }\end{array}$ & $\begin{array}{c}\text { Relative risk } \\
\text { Risk differ- } \\
\text { ence (\%) }\end{array}$ & $\begin{array}{c}p \text { value } \\
\text { F432 Adjustment disorders }\end{array}$ \\
L20-L30 Dermatitis and eczema & 33 & 7 & 4.71 & 2.0 \\
F40-F48 Anxiety, dissociative, stress-related, somatoform & 34 & 8 & 4.25 & 2.0 \\
$\quad$ and other nonpsychotic mental disorders & & 11 & 3.55 & 2.1 \\
F43 Reaction to severe stress, and adjustment disorders & 35 & 9 & 3.89 & 0.002 \\
L00-L99 Diseases of the skin and subcutaneous tissue & 87 & 44 & 1.98 & 3.0 \\
\hline
\end{tabular}

ICD-10 10th revision of the International Statistical Classification of Diseases and Related Health Problems 
underlying severe disease in general, rather than the pharmacologic effect of TNF- $\alpha$ inhibitors. A recent study showed that the risk of related conditions is higher in pediatric IBD patients in comparison with the general population: hazard ratios were 1.6 for mood disorders (427 events) and 1.5 for anxiety disorders (673 events), although the study did not investigate whether disease severity is a risk factor [27].

Key strengths of this study were the generalizability and large sample of pediatric patients analyzed through routinely collected healthcare data on non-selected TNF- $\alpha$ inhibitor initiators from the national Danish population. Patients were identified during a study period of 13 years and followed for 1 year on average. The data sources were also granular and comprehensive enough to allow for robust confounding control and to identify a large range of potential adverse events. However, null findings in this type of hypothesis-generating study cannot be interpreted as an absence of adverse events. Insufficient power or too scattered recording of certain diagnoses can lead to non-significant clusters.

Our use of the recently developed tree-based scan statistics enabled scanning for clusters of events at multiple levels of diagnosis granularity, for temporal clustering in relationship to drug initiation, and simultaneously adjust for multiple testing to generate valid p-values. The self-controlled and propensity score-matched analyses complemented each other. By performing both, we were able to capture signals of potential adverse events based on both temporally increased incidence and generally increased incidence in comparison with matched, no-use episodes. The lack of a priori defined potential adverse events and potential risk windows were strengths of the analysis.

A potential limitation was residual confounding. The propensity score-matched analysis was susceptible to confounding by indication and the self-controlled analysis to time-dependent confounding within TNF- $\alpha$ inhibitor users. In the propensity score-matched analysis, we adjusted for the general potential risk factors age, sex, underlying disease, disease duration, treatment history, and general healthcare and drug use. Given that disease severity, which is generally higher in TNF- $\alpha$ inhibitor users, is positively associated with the risk of many potential adverse events, it was unlikely that residual confounding by indication resulted in false negatives.

We chose not to use alternative study designs that might have decreased confounding additionally, including active comparator new user and prevalent new user designs [28, 29], due to the large exclusion of TNF- $\alpha$ inhibitor users and reduced power that these designs would have required. Given the hypothesis-generating aim of the study, we prioritized analyzing all TNF- $\alpha$ inhibitor initiators during the study period. As for all adverse event data-mining studies, we analyzed a large set of potential outcomes simultaneously and we did not adjust for specific risk factors in relation to each outcome. The included factors represent key confounders in relation to most types of outcomes-as themselves or as proxies for other factors. The aim of the analysis was to detect signals of potential adverse events, rather than inferring causality between drug and outcomes. Nonetheless, robust confounding control increases the validity of the results.

Another potential limitation of the study was misclassification of exposure or outcomes, which can lead to biased results. All TNF- $\alpha$ inhibitor treatment is administered in specialist care in Denmark, and coverage in the national patient register is considered to be high $[13,30]$. A previous study has validated the use of diagnosis codes from the Danish national patient register to detect outcomes [31]. We did not have access to general practice records, which meant that adverse events only diagnosed in the primary-care setting could not be detected in the analysis. However, children with serious and chronic disease, such as IBD and JIA, are cared for almost exclusively in specialist care.

\section{Conclusions}

This adverse event-screening study identified no previously unknown adverse events of TNF- $\alpha$ inhibitors in pediatric patients. The study also showed how newly developed methods for health-register screening can provide comprehensive and relevant adverse event signal detection. In pediatric patient groups where data are scarce, this approach can complement other types of studies in generating drug-safety evidence.

\section{Declarations}

Funding Open access funding provided by Karolinska Institute. This study was supported by the Swedish Research Council (201601974), Frimurare Barnhuset Foundation, and the Karolinska Institutet Research Foundation. Pasternak was supported by an investigator grant from the Strategic Research Area Epidemiology program at Karolinska Institutet. The funders had no role in the design and conduct of the study; collection, management, analysis, and interpretation of the data; preparation, review, or approval of the manuscript; or the decision to submit the manuscript for publication.

Conflict of interest J.F. Ludvigsson coordinates a study on behalf of the Swedish IBD quality register (SWIBREG) outside the submitted work. That study has received funding from Janssen corporation. H. Svanström reports consulting fees from Celgene and employment at IQVIA outside the submitted work. V. Wintzell, M. Melbye, B. Pasternak, and M. Kulldorff have no conflicts of interest to declare.

Ethics approval No approval was needed because this was a registerbased study.

Informed consent Not required because the analysis was based on secondary register data. 
Consent for publication All authors consented to publication.

Availability of data and material Not available.

Code availability Not available.

Author contributions VW had full access to all of the data in the study and takes responsibility for the integrity of the data and the accuracy of the data analysis. Study concept and design: VW, HS, BP, MK. Acquisition of data: BP. Analysis and interpretation of data: All authors. Drafting of the manuscript: VW. Critical revision of the manuscript for important intellectual content: All authors. Statistical analysis: VW. Obtained funding: BP. Study supervision: BP, MK.

Open Access This article is licensed under a Creative Commons Attribution-NonCommercial 4.0 International License, which permits any non-commercial use, sharing, adaptation, distribution and reproduction in any medium or format, as long as you give appropriate credit to the original author(s) and the source, provide a link to the Creative Commons licence, and indicate if changes were made. The images or other third party material in this article are included in the article's Creative Commons licence, unless indicated otherwise in a credit line to the material. If material is not included in the article's Creative Commons licence and your intended use is not permitted by statutory regulation or exceeds the permitted use, you will need to obtain permission directly from the copyright holder. To view a copy of this licence, visit http://creativecommons.org/licenses/by-nc/4.0/.

\section{References}

1. Hyams J, Crandall W, Kugathasan S, et al. Induction and maintenance infliximab therapy for the treatment of moderate-to-severe Crohn's disease in children. Gastroenterology. 2007;132(3):86373 (Quiz 1165-1166).

2. Ruemmele FM, Lachaux A, Cezard JP, et al. Efficacy of infliximab in pediatric Crohn's disease: a randomized multicenter open-label trial comparing scheduled to on demand maintenance therapy. Inflamm Bowel Dis. 2009;15(3):388-94.

3. Hyams J, Damaraju L, Blank M, et al. Induction and maintenance therapy with infliximab for children with moderate to severe ulcerative colitis. Clin Gastroenterol Hepatol. 2012;10(4):391-9 (e391).

4. Singh JA, Wells GA, Christensen R, et al. Adverse effects of biologics: a network meta-analysis and Cochrane overview. Cochrane Database Syst Rev. 2011(2):CD008794. https://doi. org/10.1002/14651858.CD008794.pub2

5. Singh JA, Cameron C, Noorbaloochi S, et al. Risk of serious infection in biological treatment of patients with rheumatoid arthritis: a systematic review and meta-analysis. Lancet. 2015;386(9990):258-65.

6. Wintzell V, Svanström H, Melbye M, et al. Use of tumour necrosis factor- $\alpha$ inhibitors and the risk of serious infection in paediatric inflammatory bowel disease in Denmark: a nationwide cohort study. Lancet Gastroenterol Hepatol. 2019;4(11):845-53.

7. Steinbrook R. Testing medications in children. N Engl J Med. 2002;347(18):1462-70.

8. Field MJ, Behrman RE, editors. Ethical conduct of clinical research involving children. National Academies Press, US; 2004.

9. Council of Canadian Academies. Improving medicines for children in Canada. Ottawa, ON: The Expert Panel on Therapeutic Products for Infants, Children, and Youth, Council of Canadian Academies; 2014.
10. Bourgeois FT, Kesselheim AS. Promoting pediatric drug research and labeling-outcomes of legislation. N Engl J. 2019;381(9):875-81.

11. Hennessy S, Strom BL. Improving postapproval drug safety surveillance: getting better information sooner. Annu Rev Pharmacol Toxicol. 2015;55:75-87.

12. Harpaz R, DuMouchel W, Shah NH, Madigan D, Ryan P, Friedman C. Novel data-mining methodologies for adverse drug event discovery and analysis. Clin Pharmacol Ther. 2012;91(6):1010-21.

13. Sundhetsstyrelsen. Fokusrapport-Overvågning af bivirkninger hos børn med autoimmune sygdomme i biologisk behandling. Sundhetsstyrelsen; 2012. https://laegemiddelstyrelsen.dk/ /media /85FC2763F2E84E7CB354E1AAF0527199.ashx

14. Austin PC. An introduction to propensity score methods for reducing the effects of confounding in observational studies. Multivariate Behav Res. 2011;46(3):399-424.

15. Kulldorff M, Fang Z, Walsh S. A tree-based scan statistic for database disease surveillance. Biometrics. 2003;59(2):323-31.

16. Wang SV, Maro JC, Baro E, et al. data mining for adverse drug events with a propensity score-matched tree-based scan statistic. Epidemiology. 2018;29(6):895-903.

17. Kulldorff M, Dashevsky I, Avery TR, et al. Drug safety data mining with a tree-based scan statistic. Pharmacoepidemiol Drug Saf. 2013;22(5):517-23.

18. Li R, Weintraub E, McNeil MM, et al. Meningococcal conjugate vaccine safety surveillance in the vaccine safety datalink using a tree-temporal scan data mining method. Pharmacoepidemiol Drug Saf. 2018;27(4):391-7.

19. Yih WK, Kulldorff M, Dashevsky I, Maro JC. Using the selfcontrolled tree-temporal scan statistic to assess the safety of live attenuated herpes zoster vaccine. Am J Epidemiol. 2019;188(7):1383-8.

20. Dwass M. Modified randomization tests for nonparametric hypotheses. Ann Math Stat. 1957;28:181-7.

21. Andrade P, Lopes S, Gaspar R, Nunes A, Magina S, Macedo G. Anti-tumor necrosis factor-alpha-induced dermatological complications in a large cohort of inflammatory bowel disease patients. Dig Dis Sci. 2018;63(3):746-54.

22. Freling E, Baumann C, Cuny JF, et al. Cumulative incidence of, risk factors for, and outcome of dermatological complications of anti-TNF therapy in inflammatory bowel disease: a 14-year experience. Am J Gastroenterol. 2015;110(8):1186-96.

23. Sridhar S, Maltz RM, Boyle B, Kim SC. Dermatological manifestations in pediatric patients with inflammatory bowel diseases on anti-TNF therapy. Inflamm Bowel Dis. 2018;24(9):2086-92.

24. Courbette $\mathrm{O}$, Aupiais C, Viala J, et al. Infliximab paradoxical psoriasis in a cohort of children with inflammatory bowel disease. $\mathbf{J}$ Pediatr Gastroenterol Nutr. 2019;69(2):189-93.

25. Hiremath G, Duffy L, Leibowitz I. Infliximab-induced psoriasis in children with inflammatory bowel disease. J Pediatr Gastroenterol Nutr. 2011;52(2):230-2.

26. Malkonen T, Wikstrom A, Heiskanen K, et al. Skin reactions during anti-TNFalpha therapy for pediatric inflammatory bowel disease: a 2-year prospective study. Inflamm Bowel Dis. 2014;20(8):1309-15.

27. Butwicka A, Olen O, Larsson H, et al. Association of childhoodonset inflammatory bowel disease with risk of psychiatric disorders and suicide attempt. JAMA Pediatr. 2019;173(10):969-78.

28. Lund JL, Richardson DB, Sturmer T. The active comparator, new user study design in pharmacoepidemiology: historical foundations and contemporary application. Curr Epidemiol Rep. 2015;2(4):221-8.

29. Suissa S, Moodie EE, Dell'Aniello S. Prevalent newuser cohort designs for comparative drug effect studies by 
time-conditional propensity scores. Pharmacoepidemiol Drug Saf. 2017;26(4):459-68.

30. Larsen L, Jensen MD, Larsen MD, et al. The Danish National Registry for biological therapy in inflammatory bowel disease. Clin Epidemiol. 2016;8:607-12.
31. Schmidt M, Schmidt SA, Sandegaard JL, Ehrenstein V, Pedersen L, Sorensen HT. The Danish National Patient Registry: a review of content, data quality, and research potential. Clin Epidemiol. 2015;7:449-90.

\section{Affiliations}

\section{Viktor Wintzell $^{1}$ (D) $\cdot$ Henrik Svanström ${ }^{1,2} \cdot$ Mads Melbye $^{2,3,4} \cdot$ Jonas F. Ludvigsson ${ }^{5,6,7,8} \cdot$ Björn Pasternak $^{1,2}$. Martin Kulldorff}

1 Clinical Epidemiology Division T2, Department of Medicine Solna, Karolinska Institutet, 17176 Stockholm, Sweden

2 Department of Epidemiology Research, Statens Serum Institut, Copenhagen, Denmark

3 Department of Clinical Medicine, University of Copenhagen, Copenhagen, Denmark

4 Department of Medicine, Stanford University School of Medicine, Stanford, CA, USA

5 Department Medical Epidemiology and Biostatistics, Karolinska Institutet, Stockholm, Sweden
6 Department of Pediatrics, Örebro University Hospital, Örebro University, Örebro, Sweden

7 Division of Epidemiology and Public Health, School of Medicine, University of Nottingham, Nottingham, UK

8 Department of Medicine, Columbia University College of Physicians and Surgeons, New York, NY, USA

9 Division of Pharmacoepidemiology and Pharmacoeconomics, Department of Medicine, Harvard Medical School and Brigham and Women's Hospital, Boston, MA, USA 